\title{
A GENERALIZATION OF THE CARTAN- BRAUER-HUA THEOREM
}

\author{
EUGENE SCHENKMAN AND W. R. SCOTT
}

Let $K$ be a division ring, $K^{\prime}$ its multiplicative group, and $M$ a subdivision ring. In a recent note, Schenkman [3] proved that if $M$ is a subfield whose multiplicative group $M^{\prime}$ is subinvariant in $K^{\prime}$, then either $M=K$ or $M$ is contained in the center of $K$. For the case of noncommutative $M$, the same result was obtained provided $M$ contains at least five elements of the center of $K$. However, there is a gap in the proof for the noncommutative case. ${ }^{1}$ It is the purpose of this paper to fill this gap and to remove the restriction on $M$ in the noncommutative case. Combining the result here with the result in [3], we will have

THEOREM. The only subinvariant subdivision rings of a division ring $K$ are $K$ itself and subfields of the center of $K$.

If $S$ is a subset of $K$, then $Z(S)$ will denote its centralizer in $K$ and $N(S)$ its normalizer in $K^{\prime}$. For brevity, $N(N(S)$ ) will be written $N^{2}(S)$. The notation $J \triangle L$ means that $J$ is an invariant subgroup of $L$. A subgroup or subdivision ring $M_{1}$ is a conjugate of $M$ via $H$ if there is an $x \in H$ such that $M_{1}=M^{x}=x^{-1} M x$. If $A$ and $B$ are sets, then $A<B$ means that $A$ is a proper subset of $B$, while $B \backslash A$ denotes the complement of $A$ in $B$.

For convenience, Lemmas 1 and 2 of [3] will be restated here.

Lemma 1. If $M$ is a subdivision ring of a division ring $K, x \in N(M)$, $x \notin M, x \notin Z(M), m \in Z(M) \cap M^{\prime}$, then $m+x \notin N(M)$.

Lemma 2. If $H$ and $M$ are subdivision rings of $K, H \nsubseteq M, H^{\prime} \subset N(M)$, then $H \subset Z(M)$.

Lemma $3{ }^{2}$ Let $L$ and $M$ be subdivision rings of $K$ with $L$ noncommutative. Then the index $\left[L^{\prime}: L^{\prime} \cap N(M)\right] \neq 2$.

Proof. Let $P=L \cap M, Q=L \cap Z(M), R=L^{\prime} \cap N(M)$. Then $P^{\prime} \triangle R$ and $Q^{\prime} \triangle R$. Now deny the lemma. Assume that $x \in R, x \notin M$, $x \in Z(M)$. By Lemma $1, x+1 \notin N(M)$ and $x-1 \notin N(M)$. Since

Presented to the Society, January 29, 1960 ; received by the editors August 1, 1959.

1 The gap occurs in the first half of the next to the last sentence in the paper, where the case $N(M)=N^{2}(M)$ is not considered.

2 For closely related results, see Faith [1, Theorem 3 and Corollary 1$]$. 
$\left[L^{\prime}: R\right]=2, x^{2}-1=(x+1)(x-1) \in R$. Since $x^{2} \in N(M)$, it follows from Lemma 1 that $x^{2} \in M$ or $x^{2} \in Z(M)$, and so $x^{2} \in P$ or $x^{2} \in Q$. Thus for any $y \in R, y^{2} \in P$ or $y^{2} \in Q$. It follows that

(1) If $u \in L$, then $u^{4} \in P$ or $u^{4} \in Q$.

We assert that

(2) There is a function $f$ from $L$ to the natural numbers such that either (i) $u^{f(u)} \in P$ for all $u \in L$, or (ii) $u^{f(u)} \in Q$ for all $u \in L$.

In fact, if (2) is false, there are elements $u$ and $v$ of $L$ such that $u^{n} \notin Q, v^{n} \notin P$ for all natural numbers $n$. But then by (1), $u^{4} \in P$, $v^{4} \in Q$, and by the definitions of $P$ and $Q, u^{4}$ and $v^{4}$ permute. By (1), $\left(u^{4} v^{4}\right)^{4} \in P$ or $Q$. Hence $v^{16} \in P$ or $u^{16} \in Q$, a contradiction. Hence (2) holds.

In either case of (2), there is a proper subdivision ring $S$ (one of the rings $P$ or $Q$ ) of $L$ such that $u^{f(u)} \in S$ for all $u \in L$, and such that $S$ has just one or two conjugates via $L$, namely $S$ and (perhaps) $S^{*}$. Clearly there is a function $g$ from $L$ to the natural numbers such that $u^{g(u)} \in S^{*}$ for all $u \in L$. Since $\left(S \cap S^{*}\right)^{\prime} \triangle L^{\prime}$, by the Cartan-BrauerHua theorem, $S \cap S^{*} \subset Z(L)$. Therefore $u^{f(u) \cdot(u)} \in Z(L)$ for all $u \in L$. By [2], $L$ is commutative, a contradiction.

LemMa 4. If $K$ is a division ring and $M$ a noncommutative proper subdivision ring, then $M^{\prime}$ is not subinvariant in $K^{\prime}$.

Proof. Deny the lemma. Let $n$ be the smallest natural number such that there exist a division ring $K$, a noncommutative subdivision ring $M$ and subgroups $G_{1}, \cdots, G_{n}$ such that $M^{\prime} \triangle G_{1} \triangle G_{2} \triangle \cdots$ $\triangle G_{n}=K^{\prime}$.

If $x \in K^{\prime}$ and $M<M^{x}$, then $M^{\prime} \triangle\left(G_{1} \cap M^{x}\right) \triangle \cdots \Delta\left(G_{n-1} \cap M^{x}\right)$ $=\left(M^{x}\right)^{\prime}$, contradicting the minimality property of $n$. Hence $M \nless M^{x}$ if $x \in K^{\prime}$.

By Lemma 2, if $x \in G_{2}$ and $M^{x} \neq M$, then $M \subset Z\left(M^{x}\right)$. By the Cartan-Brauer-Hua theorem, $n \neq 1$. Hence by the minimality property of $n$, there is a conjugate $M^{y} \neq M$ of $M$ with $y \in G_{2}$. Let $Q$ be the intersection of all centralizers of conjugates of $M$ via $G_{2}$, other than $M$ and $M^{y}$, and let $Q=K$ if there are no such conjugates. Then $M \subset Q$ and $M^{y} \subset Q$. It is then clear that $M$ has at most two conjugates via $G_{2} \cap Q$. Now $M^{\prime} \triangle G_{1} \cap Q \triangle \cdots \triangle G_{n} \cap Q=Q^{\prime}$. Hence, returning to the original notation, it may be assumed that $M$ has just two conjugates via $G_{2}$, namely $M$ and $M^{y}$.

If $M<Z\left(M^{y}\right)$, then $M$ is the only conjugate of itself via $G_{2} \cap Z\left(M^{y}\right)$, and the minimality of $n$ is contradicted in the usual way. Hence $M=Z\left(M^{y}\right)$, and similarly $M^{y}=Z(M)$. If $x \in N(M)$, then $M^{y x}=(Z(M))^{x}$ $=Z\left(M^{x}\right)=Z(M)=M^{y}$, so that $N\left(M^{y}\right) \supset N(M)$. Similarly $N(M)$ 
$\supset N\left(M^{y}\right)$. Hence $N\left(M^{y}\right)=N(M)$. Therefore $N(M)^{y}=N\left(M^{y}\right)=N(M)$, and $y \in N^{2}(M) \backslash N(M)$.

If $x \in G_{2}$, then $M^{x}=M$ or $M^{y}$, so that $x \in N(M)$ or $x \in N(M) y$. Hence $G_{2} \subset N^{2}(M)$. Now if $G_{2}=K^{\prime}$, then $M$ has just two conjugates in $K$, in contradiction to Lemma 3. Thus $G_{2}<K^{\prime}$. If $G_{3} \subset N^{2}(M)$, then $M^{\prime} \triangle G_{3} \cap N(M) \triangle G_{3} \cap N^{2}(M)=G_{3}$, contradicting the minimality of $n$. Hence there is a $u \in G_{3} \backslash N^{2}(M)$. Then $\left(M^{u}\right)^{\prime} \subset G_{2} \subset N^{2}(M)$. If $M^{u} \nsubseteq N(M)$, then $\left[\left(M^{u}\right)^{\prime}: M^{u} \cap N(M)\right]=2$. This contradicts Lemma 3. Therefore $\left(M^{u}\right)^{\prime} \subset N(M)$. Since $u \notin N^{2}(M), M^{u} \neq M$ or $M^{y}$. By Lemma $2, M^{u}<Z(M)=M^{y}$. Thus $M<M^{y^{u}}{ }^{-1}$, which was shown to be impossible in the first part of the proof.

\section{BIBLIOGRAPHY}

1. C. C. Faith, On conjugates in division rings, Canad. J. Math. vol. 10 (1958) pp 374-380.

2. I. Kaplansky, $A$ theorem on division rings, Canad. J. Math. vol. 3 (1951) pp. 290-292.

3. Eugene Schenkman, Some remarks on the multiplicative group of a sfield, Proc. Amer. Math. Soc. vol. 9 (1958) pp. 231-235.

UNIVERSITY OF WISCONSIN,

Louisiana State University and

UNIVERSITY OF KANSAS 